\title{
Cavity modes in one-dimensional photonic crystal slabs
}

\author{
D. GERACE${ }^{1}$, M. A G I O ${ }^{1}$, L.C.A N D R E A N I ${ }^{1}$ A N D P. L A L A N N E \\ ${ }^{1}$ Istituto Nazionale per la Fisica della Materia and Dipartimento di Fisica "Alessandro Volta," Università di \\ Pavia, via Bassi 6, I-27100 Pavia, Italy \\ ${ }^{2}$ Laboratoire Charles Fabry de l'Institut de Optique, Centre National de la Recherche Scientifique, BP 147, \\ F-91403, Orsay Cedex, France \\ (E-mail: gerace@fisicavolta.unipv.it)
}

\begin{abstract}
A theoretical study of cavity modes in one-dimensional photonic crystal slabs embedded in Silicon-on-Insulator structures is reported. Three different methods are employed, namely a guided-mode expansion in which the coupling to radiative modes is treated by perturbation theory, a grating or scattering-matrix method for calculating the surface reflectance, and a Fourier modal expansion for inplane transmission calculations. It is shown that all methods lead to the same values for the quality factors of cavity modes for both first- and second-order Bragg mirrors. We conclude that the quality factor of a cavity mode can be determined with optical reflectance from the surface of the slab.
\end{abstract}

Key words: optical waveguides, photonic crystals, Fabry-Pérot cavities

\section{Introduction}

Photonic crystals $(\mathrm{PhC})$ embedded in planar dielectric waveguides, commonly known as photonic crystal slabs, are of great interest for the control of the electromagnetic field at optical wavelengths (Johnson et al. 1999; Sakoda 2001; Johnson and Joannopoulos 2002). In particular, systems characterized by a strong dielectric modulation in the plane of the waveguide and a high dielectric contrast between the core layer and the upper and lower claddings have been receiving much attention owing to the in-plane control provided by the photonic band gap and to the vertical confinement of light induced by dielectric mismatch. These systems are characterized by the existence of truly guided modes lying below the light line of the cladding material (or materials if the waveguide is asymmetric), and of quasi-guided modes if their energy lies above the light line in the first Brillouin zone. The latter are coupled to the radiative modes of the planar waveguide, thus producing out-of-plane diffraction losses and a related spectral linewidth in the optical excitation of these resonances; quasi-guided modes can be excited by plane waves incident from the surface of the slab (Popov 1993; Fujita et al. 1998; Astratov et al. 1999). On the contrary, truly guided modes have zero intrinsic linewidth and can be excited by in-plane 
transmission measurements, or else by attenuated total reflectance using a prism as recently reported in (Galli et al. 2004). It is very difficult to treat the electromagnetic problem in these systems from a general point of view, i.e. by using a single theoretical approach that allows to calculate all the physical properties, such as complex photonic dispersion, reflection or transmission and losses, in a three-dimensional theoretical scheme. Exact numerical methods such as finite-difference-time-domain (FDTD) can in principle give a complete description of the problem, but they are rather heavy from a computational point of view, which limits also the physical dimensions of the systems that can be modelled. Alternative theoretical approaches employed to study one-dimensionally patterned waveguide gratings, such as the eigenmode expansion method, have also been used in the literature (Čtyroký et al. 2002).

In this work we study defect cavities in one-dimensional (1D) PhC slabs, namely Fabry-Pérot cavities in waveguide geometry, and address the problem of determining the quality (Q) factor of localized cavity modes. These devices are considered as potential building blocks in Silicon-On-Insulator (SOI) planar photonic integrated circuits operating at optical wavelengths for advanced telecom applications, thus we focus on SOI slab structures. We consider defect cavities surrounded by first- and second-order Bragg mirrors. We tackle the problem by three different theoretical approaches: (i) a recently developed guided-mode expansion (GME) method (Andreani and Agio 2002; Andreani 2002) that yields the mode energies and $Q$-factors, (ii) a calculation of surface reflectance at varying angles of incidence using grating or scattering-matrix methods (SMM), and (iii) a calculation of in-plane transmission on a finite structure employing a Fourier modal expansion (FME) method. The GME and SMM methods require that the defect cavity be repeated with supercell periodicity along the 1D axis, while the FME method deals with a finite structure. The goal of this work is twofold. First, to show that the different methods yield the same results for the $Q$-factors. Second, to prove that the intrinsic $Q$-factor of a single cavity can be determined by working on a periodic structure in a diffraction geometry, i.e., by measuring the reflectance from the surface of the sample at varying angles of incidence.

This paper is organized as follows. The theoretical methods are briefly reviewed in Section 2, in particular we shall discuss the structure and the physical quantities addressed by each specific method. In Section 3 we present and discuss the results concerning the structure under study and the conditions leading to the determination of $Q$-factors of cavity modes. Section 4 contains concluding remarks. 


\section{Overview of theoretical methods}

\subsection{THE GUIDED-MODE EXPANSION}

This method has been recently proposed to study guided and quasi-guided modes in PhC slabs (Andreani and Agio 2002; Andreani 2002) and it is presented here in its general formulation. Starting from the second-order Maxwell equation for the magnetic field

$$
\nabla \times\left[\frac{1}{\epsilon(\mathbf{r})} \nabla \times \mathbf{H}\right]=\frac{\omega^{2}}{c^{2}} \mathbf{H}
$$

where $\epsilon(\mathbf{r})$ is the spatially dependent dielectric constant, the field is expanded in an orthonormal set of basis states as

$$
\mathbf{H}(\mathbf{r})=\sum_{\mu} c_{\mu} \mathbf{H}_{\mu}(\mathbf{r})
$$

and then Equation (1) is transformed into a linear eigenvalue problem

$$
\sum_{v} \mathcal{H}_{\mu \nu} c_{v}=\frac{\omega^{2}}{c^{2}} c_{\mu}
$$

where the matrix $\mathcal{H}_{\mu v}$ is given by

$$
\mathcal{H}_{\mu v}=\int \frac{1}{\epsilon(\mathbf{r})}\left[\nabla \times \mathbf{H}_{\mu}^{*}(\mathbf{r})\right] \cdot\left[\nabla \times \mathbf{H}_{v}(\mathbf{r})\right] \mathrm{d} \mathbf{r}
$$

For the case of a PhC slab, we have a planar waveguide along $z$ and a periodic 2D patterning in the $x y$ plane. The basis set $\mathbf{H}_{\mu}(\mathbf{r})$ is chosen to consist of the guided modes of an effective waveguide, where each layer $j$ (with $j=1,2,3$ ) is taken to have a homogeneous dielectric constant given by the spatial average of $\epsilon_{j}(x, y)$ within the unit cell. It should be noted that the definition of the effective dielectric constant as a spatial average is not unique. For the effective homogeneous waveguide the planar dynamics can be separated from the vertical one, thus the index $\mu$ can be written as $\mu=(\mathbf{k}+\mathbf{G}, \alpha)$, where $\mathbf{k}$ is the Bloch vector in the $x y$ plane, $\mathbf{G}$ is a $2 \mathrm{D}$ reciprocal lattice vector and $\alpha=1,2, \ldots, \alpha_{\max }$ is a discrete index which labels the guided modes at wave vector $\mathbf{k}+\mathbf{G}$. The magnetic field is then written as

$$
\mathbf{H}_{\mathbf{k}}(\mathbf{r})=\sum_{\mathbf{G}} \sum_{\alpha<\alpha_{\max }} c_{\alpha}(\mathbf{k}+\mathbf{G}) \hat{\mathbf{F}}_{\alpha}(z) \mathrm{e}^{i(\mathbf{k}+\mathbf{G}) \cdot \mathbf{x}}
$$


where $\hat{\mathbf{F}}_{\alpha}(z)$ is a normalized spatial envelope function representing the guided mode of the effective waveguide (Yariv and Yeh 1984) and $\mathbf{x}=(x, y)$ is the inplane spatial vector. Equation (4) then becomes

$$
\mathcal{H}_{\mathbf{G}, \mathbf{G}^{\prime}}^{\alpha, \alpha^{\prime}}=\int \frac{1}{\epsilon(\mathbf{r})}\left[\nabla \times \hat{\mathbf{F}}_{\alpha}^{*}(z) \mathrm{e}^{-i(\mathbf{k}+\mathbf{G}) \cdot \mathbf{x}}\right] \cdot\left[\nabla \times \hat{\mathbf{F}}_{\alpha^{\prime}}(z) \mathrm{e}^{i\left(\mathbf{k}+\mathbf{G}^{\prime}\right) \cdot \mathbf{x}}\right] \mathrm{d} \mathbf{r}
$$

The matrix elements of Equation (6) can be expressed in terms of the inverse dielectric tensor in each layer $\eta_{j}\left(\mathbf{G}, \mathbf{G}^{\prime}\right)=\epsilon_{j}^{-1}\left(\mathbf{G}, \mathbf{G}^{\prime}\right)$, by assuming the separability of $\epsilon(\mathbf{r})$ and calculating the 2D integral in the $x y$ plane. The matrix $\eta_{j}\left(\mathbf{G}, \mathbf{G}^{\prime}\right)$ is the same quantity which appears in usual 2D plane-wave calculations and can be conveniently evaluated by a numerical inversion of the dielectric matrix $\epsilon_{j}\left(\mathbf{G}, \mathbf{G}^{\prime}\right)$ (Ho et al. 1990). Both transverse electric (TE) and transverse magnetic (TM) guided modes are included in the expansion and are coupled by the dielectric modulation in the $x y$ plane (Ochiai and Sakoda 2001a, b), leading to the folding and splitting of guided modes in the first Brillouin zone. In the present case of a $1 D$ lattice, as schematically shown in Fig. 1a, TE and TM polarizations are uncoupled. A systematic study of photonic mode dispersion and gap maps of 1D lattices in a PhC slab has recently been performed (Gerace and Andreani 2004a). In this work only TE polarization is considered and defect cavities are treated by introducing a supercell periodicity along the $1 \mathrm{D}$ axis, as discussed in Section 3.

We point out that the guided modes of the effective waveguide represent an orthonormal set of states, however the basis set is not complete since the radiative modes are not included. The coupling to radiative modes at all energies produces a second-order shift of the resonance energies: neglecting this effect (usually of the order of a few percent) is the main approximation of the present method. A most important effect is the first-order coupling to radiative modes at the same energy for modes that fall above the light line leading to a radiative decay, i.e., to an imaginary part of the energy, which
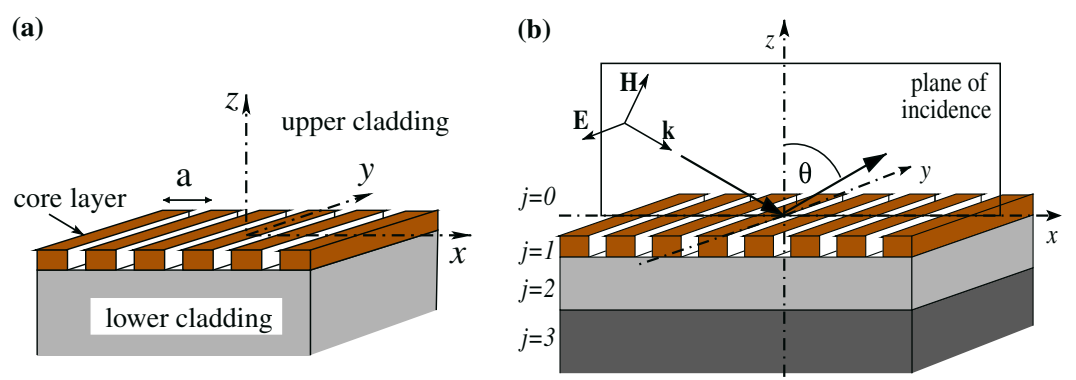

Fig. 1. Schematic illustration of (a) the three layer geometry used in the GME method; (b) the geometry for the grating method in classical diffraction and TE polarization. 
can be calculated by time-dependent perturbation theory. This procedure, formally analogous to Fermi Golden Rule in quantum mechanics, leads to an expression for the imaginary part of the frequency given by

$$
-\operatorname{Im}\left(\frac{\omega_{k}^{2}}{c^{2}}\right)=\pi\left|\mathcal{H}_{\text {leaky,guided }}\right|^{2} \rho\left(\mathbf{k} ; \frac{\omega_{k}^{2}}{c^{2}}\right)
$$

where $\rho\left(\mathbf{k} ; \omega_{k}^{2} / c^{2}\right)$ is the 1D density of photonic states at fixed in-plane wave vector (Ochiai and Sakoda 2001b), and the operator $\mathcal{H}_{\text {leaky,guided }}$ is expressed as

$$
\mathcal{H}_{\text {leaky,guided }}=\int \frac{1}{\epsilon(\mathbf{r})}\left[\nabla \times \mathbf{H}_{\text {leaky }}^{*}(\mathbf{r})\right] \cdot\left[\nabla \times \mathbf{H}_{\text {guided }}(\mathbf{r})\right] \mathrm{d} \mathbf{r}
$$

Results for intrinsic losses in 1D PhC slabs and for intrinsic and extrinsic (disorder induced) losses in 2D PhC slabs with line defects have been presented elsewhere (Andreani and Agio 2003; Gerace and Andreani 2004a, b).

Besides neglecting the second order coupling to radiative modes, the main approximations of the present method concern the choice of the average dielectric constant in each layer, which defines the basis states for the expansion, and the truncation of the reciprocal lattice vectors to a set with modulus lower than a certain $\left|\mathbf{G}_{\max }\right|$. The number of guided modes in the expansion is usually taken to be $\alpha_{\max }=8$ in the calculations, which is largely sufficient for convergence in the energy range considered. The momentumspace cutoff plays the same role and has the same properties as in usual plane-wave calculations.

\subsection{THE GRATING METHOD}

The $Q$-factor of cavity modes studied in Section 3 can also be estimated by considering a periodic system along the direction of the cavity and calculating optical spectra for light incidence on the surface of the PhC slab. Illuminated by an incident plane wave, the transmission or reflection spectrum of the periodized cavity (a 1D grating in fact) should present an anomaly for the resonant wavelengths. This anomaly results from a pole of the scattering matrix, which relate the Rayleigh expansion of the electromagnetic fields in the claddings (Popov 1993). The method we used for the calculation of the transmission and reflection spectra is a frequency-domain method, which relies on Fourier expansion techniques for all the electromagnetic fields quantities. Because the literature on the analysis of gratings with Fourier expansion techniques is largely documented (Popov 1993), the method is not detailed here and we assume that the reader is familiar with the rigorous coupled-wave analysis (Moharam et al. 1995), with its enhanced version for 
TM polarization and conical diffraction (Lalanne and Morris 1996) and for crossed gratings (Li 1997). The implementation we used, which, can also be applied to 2D photonic lattices, is described in (Whittaker and Culshaw 1999) and will be referred to henceforth as scattering-matrix method (SMM).

The geometry for the grating calculation is shown in Fig. 1b. The light beam is incident on the surface of the structure in direction perpendicular to the grating, i.e., a classical (non-conical) diffraction geometry is used. We assume TE-polarized light with respect to the plane of incidence, as shown in the figure. Studies of other propagation direction (conical diffraction) could also be useful but are not considered here.

\subsection{FOURIER MODAL METHOD WITH PERFECTLY MATCHED LAYERS}

Fourier expansion methods like the rigorous coupled-wave analysis (Moharam et al. 1995) or the differential method (Popov and Nevière 2000), which are widely used for modelling periodic structures, can also be used for modelling non-periodic systems. Such an extension has been first pointed out in (Lalanne and Silberstein 2000; Silberstein et al. 2001). This approach drastically extends the domain of application of grating theories, and will be referred to henceforth as Fourier modal expansion (FME) method. Referring to Fig. 2, a supercell is introduced in the $z$-direction, perpendicular to the plane $x y$ of the slab. The boundaries of the supercell incorporate perfectly matched layers (Berenger 1994) that absorb the light scattered by the corrugation. The electromagnetic fields are null on every transversal boundary and can be expanded in a Fourier series (plane-wave expansion). Dividing the

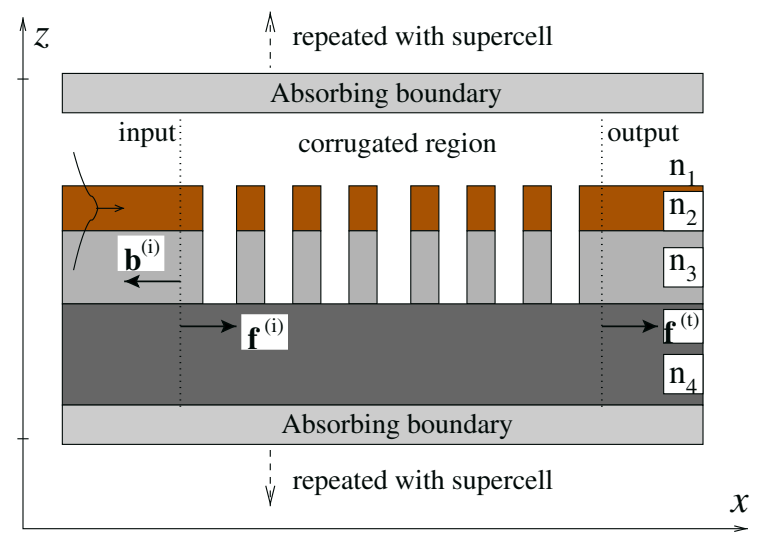

Fig. 2. Schematic picture of geometrical structure employed in the FME method. The scattering matrix is used to relate the field amplitudes between the different layers along $x$, and a Fourier expansion is made along $z$ with supercell periodicity. 
structure into uniform layers along $x$, radiative and guided modes are calculated for each layer in a Fourier basis. Transmission and reflection can be evaluated by using a scattering matrix to match the field amplitudes between different layers

$$
\left[\begin{array}{l}
\mathbf{b}^{(i)} \\
\mathbf{f}^{(i)}
\end{array}\right]=\mathbf{S}\left[\begin{array}{c}
\mathbf{0} \\
\mathbf{f}^{(t)}
\end{array}\right]
$$

where $\mathbf{b}^{(i)}$ and $\mathbf{f}^{(i)}$ are column vectors whose elements represent the amplitudes of the backward and forward propagating modes at the input plane, and similarly $\mathbf{f}^{(t)}$ is the vector of amplitudes of the forward field at the output plane. As an example, let us consider the waveguide geometry of Fig. 2. If we assume to illuminate the corrugated region with the fundamental TE mode, all components of the vector $\mathbf{f}^{(i)}$ will be zero except the component $f_{\mathrm{p}}^{(i)}=1$ corresponding to that mode. The amplitudes of backward- and forwardpropagating modes are then computed by using the S-matrix, and the reflected and transmitted intensity can be simply defined by $R=\left|b_{\mathrm{p}}^{(i)}\right|^{2}$ and $T=\left|f_{\mathrm{p}}^{(t)}\right|^{2}$. The $Q$-factor of a cavity mode can be evaluated by $Q=\operatorname{Re}(\tilde{\lambda}) /[2 \operatorname{Im}(\tilde{\lambda})]$, where $\tilde{\lambda}$ is the complex pole of the scattering matrix.

For two-dimensional geometries in integrated optics, the FME with perfectly matched layers has been benchmarked for scattering problems in dielectric Bragg mirrors (Čtyroký et al. 2002) and has been used to compute efficiently Bloch waves of grating waveguides (Cao et al. 2002). For more computationally intensive three-dimensional geometries, it has been applied to the calculation of radiation losses in line-defect $\mathrm{PhC}$ waveguides (Lalanne 2002; Sauvan et al. 2003), and to the electromagnetic analysis of optical microcavities like micropillars (Lalanne et al. 2004a) or PhC wires (Lalanne et al. 2004b).

\section{Numerical results}

We apply the theoretical tools described in Section 2 to a Fabry-Pérot cavity embedded in a SOI-based 1D PhC slab. A schematic picture of the structure is shown in Fig. 3, where the parameters are also defined and the supercell along the direction of periodicity is shown. The latter is used in the calculation of the photonic band dispersion by the GME method, and also of reflectance by the SMM.

The cavities are designed with the FME to have a resonant wavelength of the defect mode at $\lambda=1.5 \mu \mathrm{m}$ in a SOI slab with a silicon layer of thickness $d=260 \mathrm{~nm}$. Two kinds of mirrors with a mid-gap frequency equal to the resonant frequency are considered, namely first- and second-order Bragg 


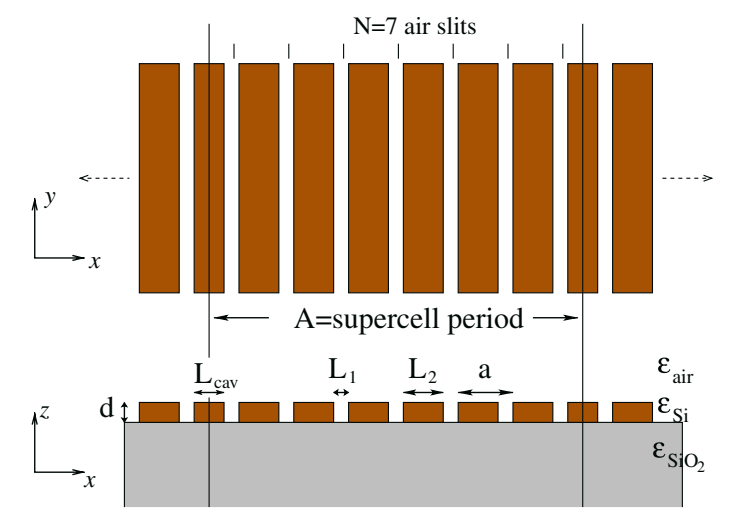

Fig. 3. Schematic picture of the structure considered for calculations with GME and SMM approaches. Upper and lower claddings are assumed to be semi-infinite along $z$, and the structure is uniform along $y$. The main structural parameters are defined. The air fraction is $f_{\text {air }}=L_{1} / a ; N$ is the number of air slits between consecutive cavity layers.

mirrors. Two physical mechanisms are responsible for the finite mode lifetime of the cavity. For first-order Bragg mirrors, the fundamental Bloch mode of the mirror is truly guided, and the mode lifetime is limited solely by a modeprofile mismatch problem between the fundamental guided mode of the slab waveguide (which is cycling between the mirrors) and the fundamental Bloch mode of the mirror (Palamaru and Lalanne 2001). This mismatch problem results into radiation losses in the claddings with a finite modal reflectivity of $98 \%$ at $\lambda=1.5 \mu \mathrm{m}$ for the fundamental guided mode impinging onto a semiinfinite mirror. The first-order gap lies fully below the cladding light lines and the intrinsic $Q$-factor calculated for a cavity with semi-infinite mirrors is rather high. The cavity designed with second-order Bragg mirrors suffers from additional losses. In this case, the second-order photonic gap lies above the light line in air and the cavity mode has sizeable contributions from leaky Bloch modes of the mirrors. Thus as the light is reflected back from the mirrors, it is additionally radiated into the claddings. This additional source of radiation results in a lower modal reflectivity of $93.5 \%$ at mid-gap frequency and to a smaller intrinsic $Q$-factor. Bragg mirrors if third and higher orders have lower $Q$-factors due to the increase of intrinsic losses with increasing frequency, as we have verified.

We start with the case of a cavity surrounded by second-order Bragg mirrors. The structure has the following parameters: $d=260 \mathrm{~nm}$, $a=560 \mathrm{~nm}, L_{\mathrm{cav}}=335 \mathrm{~nm}, L_{1}=100 \mathrm{~nm}, L_{2}=460 \mathrm{~nm}$, with an air fraction $f_{\text {air }}=L_{1} / a=0.1786$. In Fig. 4 the photonic band dispersion is shown for the ideally infinite 1D lattice as well as for the cavity structure. The frequency is plotted as a function of the component of the wave vector $\mathbf{k}$ along the periodicity direction. Only TE-polarized modes are considered here, which are the ones whose electric field is parallel to the stripes (polarization along $y$ ). 

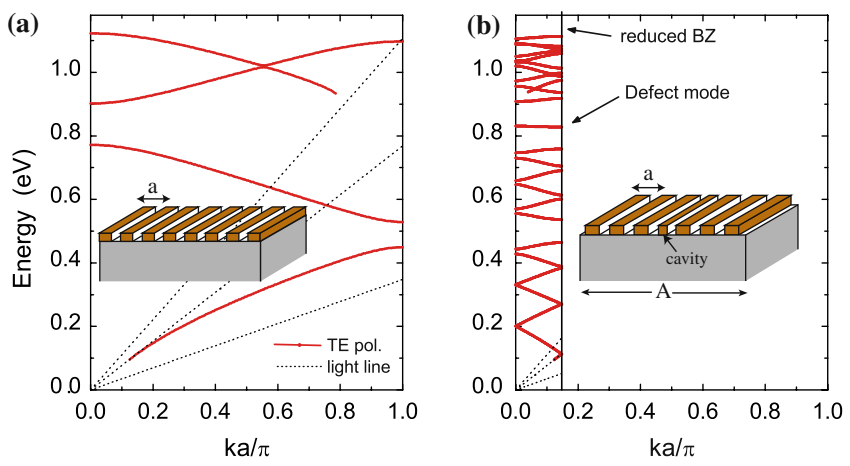

Fig. 4. Photonic band dispersion of TE-polarized modes as a function of dimensionless wave vector for (a) 1D lattice of Silicon stripes on a $\mathrm{SiO}_{2}$ cladding with lattice constant $a=560 \mathrm{~nm}$, thickness $d=260 \mathrm{~nm}$, air fraction $f_{\text {air }}=0.1786$, and (b) $1 \mathrm{D}$ cavity of length $L_{\mathrm{cav}}=335 \mathrm{~nm}$ and seven air slits between two consecutive cavities in the supercell.

The dielectric constants of the patterned core layer and the uniform upper and lower claddings are set to the following values: $\epsilon_{\mathrm{air}}=1, \epsilon_{\mathrm{Si}}=12.1104$, $\epsilon_{\mathrm{SiO}_{2}}=2.0736$, as appropriate for these materials around $\lambda=1.5 \mu \mathrm{m}$. An effective dielectric constant given by the spatial average in each layer is chosen to define the basis set for the GME method, which is a very good approximation for TE modes (Gerace and Andreani 2004a). The three light lines appearing in Fig. 4 correspond, for increasing energies, to the effective core layer with average dielectric constant $\epsilon_{\text {eff }}$, to the uniform $\mathrm{SiO}_{2}$ cladding and to the air light line. The dispersion of the Fabry-Pérot resonator is displayed in a reduced Brillouin zone (BZ) in Fig. 4b, owing to the superperiodicity introduced in the calculation. The supercell period is defined as $A=N L_{1}+(N-1) L_{2}+L_{\mathrm{cav}}=N a-L_{2}+L_{\mathrm{cav}}$, and it is about seven times larger than the period of the Bragg reflector, $a$, in this particular case; thus, the $\mathrm{BZ}$ is reduced by a factor of about seven. The photonic bands, folded in the reduced zone, can be recognized in Fig. $4 \mathrm{~b}$ as compared to those of Fig. 4a. The main difference is that a defect mode appears as an almost dispersionless band within the TE band gap, at an energy $E_{\text {cav }} \simeq 0.825 \mathrm{eV}$ $(\lambda=1.5 \mu \mathrm{m})$. This defect mode lies in the radiative region of the $(\mathbf{k}, \omega)$ plane and it can be excited by light incident on the surface of the planar waveguide (Bristow et al. 2003).

In Fig. 5a the band dispersion of the defect mode is displayed in the energy range between 0.7 and $1 \mathrm{eV}$, and in Fig. 5(b) the imaginary part of mode energies calculated by the perturbative approach described in Section 2.1 is also shown. The imaginary part of frequency is always much smaller than the real part, thereby justifying a posteriori the perturbative treatment. In Fig. 5c the calculation of variable angle reflectance by the SMM is plotted on the same energy range, in order to make a direct comparison with the dispersion of Fig. 5a and b. The same supercell along the periodicity direction was 

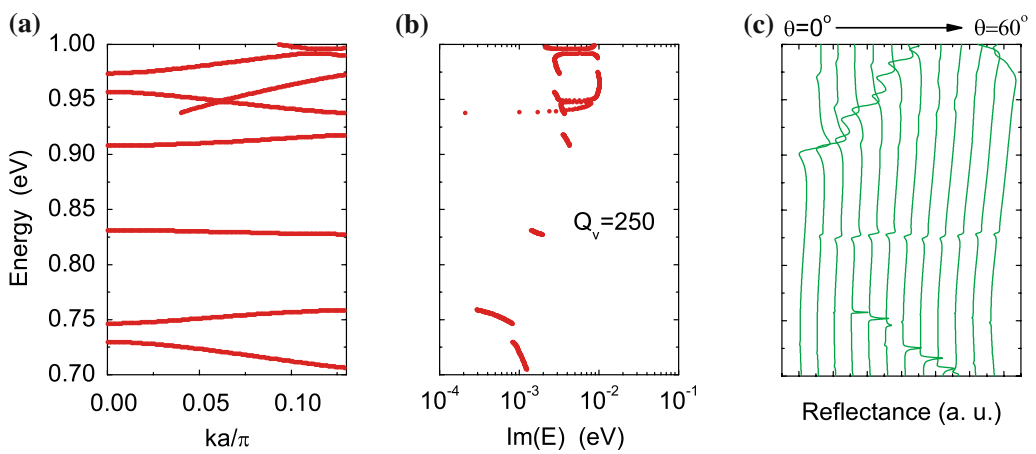

Fig. 5. (a) Dispersion of the defect mode, (b) imaginary part of mode energy corresponding to each photonic band, and (c) surface reflectance for angles ranging from $0^{\circ}$ to $60^{\circ}$ in steps of $5^{\circ}$ for classical diffraction and TE polarization (geometry of Fig. 1b). Reflectance curves are shifted by $\Delta R=0.5$ for clarity. Structure parameters are the same as in Fig. 4.

employed for SMM calculations. It is known (Popov 1993; Fujita et al. 1998; Astratov et al. 1999) that the sharp resonance features observed in reflectance or transmittance experiments correspond to the excitation of photonic modes which are matched in frequency and wave vector to the incoming beam. This way, each resonance marks a point in the $(\mathbf{k}, \omega)$ plane, and the wave vector component parallel to the surface can be extracted from the angle of incidence by the relation $k=(\omega / c) \sin \theta$. The SMM calculation allows to reproduce exactly the experimental situation described above. Very good agreement between GME and SMM is found in the present case, in particular concerning the energy position of the defect mode and of the band gap edges. The excitation of the cavity mode can be recognized in the reflectance spectrum as a dispersionless feature as a function of the angle of incidence. Moreover, the spectral linewidth of the structure observed in reflectance are related to the imaginary parts of Fig. 5b. Experimental results on SOI-based 1D PhC slabs have been reported (Patrini et al. 2002), in which the photonic band dispersion of quasi-guided modes is obtained by variable angle reflectance measurements. Moreover, cavity modes of Fabry-Pérot resonators with supercell periodicity were probed with the same experimental technique on GaAs-based systems (Bristow et al. 2003). The vertical $Q$-factor, $Q_{\mathrm{v}}$, of the cavity mode, that is the one determined by out-of-plane losses, depends slightly on the parallel wave vector because the imaginary part of the energy does. This effect vanishes in the limit of a very large supercell. In order to smear out the effect of a finite supercell width and to get a single number for the $Q_{\mathrm{v}}$-factor from the calculations of Fig. 5a and $\mathrm{b}$, we average the mode energy and the corresponding imaginary part over the first BZ and then apply the definition $Q_{\mathrm{v}}=E_{\mathrm{av}} /[2 \operatorname{Im}(E)]_{\mathrm{av}}$. Thus, a $Q_{\mathrm{v}}$ of 250 is obtained for such a structure, which is in favorably good agreement with the $Q$-factors estimated from the resonant features in reflectance spectra. The latter do not 
change appreciably when changing angle of incidence, which is in good agreement with the calculations of Fig. 5b. For an estimation of the $Q_{\mathrm{v}}$ from reflectance spectra, we chose to analyze the resonant feature at $10^{\circ}$, which has a resonance energy of $E_{\mathrm{cav}} \simeq 0.825 \mathrm{eV}$ and an estimated full width at half maximum $(\mathrm{FWHM})$ of $\sim 3.5 \mathrm{meV}$, which roughly correspond to $Q_{\mathrm{v}} \simeq 235$. This value, obtained by dividing the resonance energy for the FWHM, slightly depends on the number of air slits in the mirrors around the cavity layer and is already converged for $N=7$.

In Fig. 6 transmission calculations by the FME method are shown for a 1D PhC slab of finite length (Fig. 6a), and for a cavity between two mirrors with the same number of slits (Fig. 6b). The mirrors are composed of five air slits in both cases. The same structure parameters as in the previous calculations are used. The reflection and transmission coefficients are calculated after excitation with the fundamental TE guided mode of the initial planar waveguide. The band gap is clearly seen to be in good quantitative agreement with both GME calculation of Fig. 5a and SMM calculations of Fig. 5c, between 0.75 and $0.9 \mathrm{eV}$. The cavity mode produces a Lorentzian peak in the transmission spectrum, which is displayed in Fig. 6b, with a calculated $Q$ factor of 188. The total Q of such a system can be expressed as (Painter et al. 1999)

$$
Q=\frac{Q_{\mathrm{p}} Q_{\mathrm{v}}}{Q_{\mathrm{p}}+Q_{\mathrm{v}}}
$$

where $Q_{\mathrm{p}}$ is the planar $Q$-factor due to the confinement induced by the mirrors. The resonant transmission in the cavity mode is very low in this case, owing to reflection and diffraction losses over the length of the structure (calculations not shown).
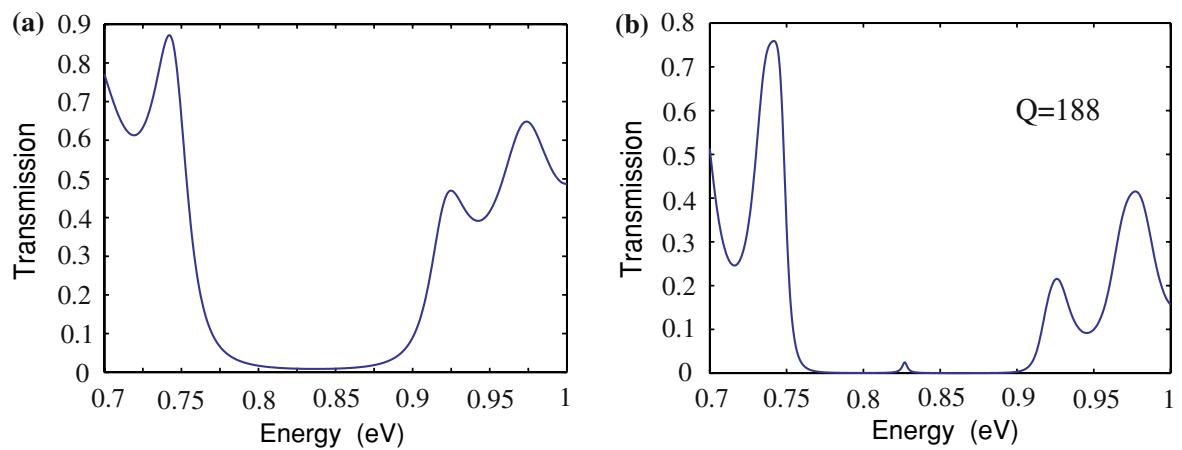

Fig. 6. (a) Transmission through five air slits Bragg mirror (parameters as in Fig. 4); the width of the photonic band gap should be compared to calculations of Fig. 5a and c. (b) Transmission through a structure analogous to the one of Fig. 7a, with a cavity surrounded by mirrors with five air slits. 
In order to make a direct comparison with the calculations of Fig. 5, we show a detailed view of the transmission peak for the structure represented in Fig. 7a. The cavity layer is inserted between two Bragg mirrors with seven air slits each. The calculated total $Q$-factor for this structure is $Q=231$, as indicated in Fig. 7b. This value is in good quantitative agreement with the one obtained by the calculation of $Q_{\mathrm{v}}$ from either the GME or the SMM methods in Fig. 5. This means that with seven air slits in the mirrors $Q_{\mathrm{p}}$ is much larger than $Q_{\mathrm{v}}$, and thus $Q \simeq Q_{\mathrm{v}}$.

In Table 1 the calculated $Q$-factors and the maxima of the transmission peaks for different numbers of air slits in the mirrors are shown. On increasing the number of periods in the mirrors surrounding the cavity, it is clear that the total $Q$-factor tends to $Q_{\mathrm{v}}$. Thus we confirm that all theoretical methods adopted here lead to the same value for the vertical $Q$-factor. Also, we conclude that the $Q$-factor can be determined by measuring the radiative coupling between a plane wave incident from the surface and the cavity mode with supercell repetition, as described by the calculations of Fig. 5. This may be more convenient than measuring the in-plane modal transmission: indeed, when the mirrors have many periods the total structure is longer in length and the transmission in the cavity mode is low, because of reflection and diffraction losses, thus making the measurement a difficult one.

We notice that no optimization of the structure was made to reach a high $Q$-factor as done, e.g., in (Lalanne and Hugonin 2003). Higher $Q$-factors can be obtained in a natural way by using first-order Bragg mirrors, i.e., with a photonic gap at the border of the BZ in the region below the cladding light lines. In Fig. 8 the GME calculations for a cavity embedded in Bragg mirrors of this kind are shown. The parameters of the structure are: $d=260 \mathrm{~nm}$, $a=310 \mathrm{~nm}, L_{\mathrm{cav}}=335 \mathrm{~nm}, L_{1}=100 \mathrm{~nm}, L_{2}=210 \mathrm{~nm}$, with an air fraction $f_{\text {air }}=L_{1} / a=0.3226$. The mirrors have a first-order photonic band gap around $0.825 \mathrm{eV}$, as displayed in the 1D lattice dispersion of Fig. $8 \mathrm{a}$, and the

(a)

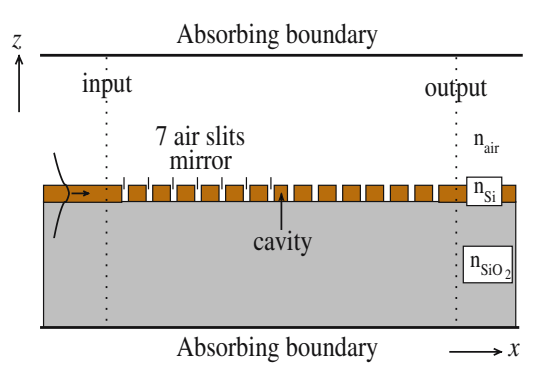

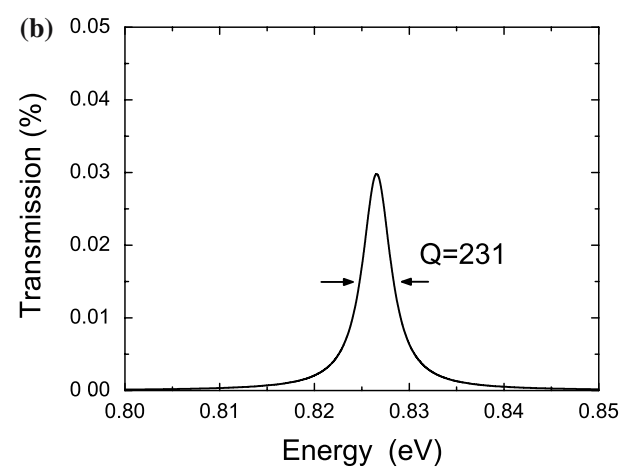

Fig. 7. (a) Schematic picture of the geometry employed for the FME calculation; (b) Transmission of the fundamental TE mode through the structures displayed in (a), with parameters as in Fig. 4. 
Table 1. Quality factors and transmission peaks calculated with the FME method

\begin{tabular}{lcc}
\hline Number of slits & $Q$-factor & $T_{\text {peak }}(\%)$ \\
\hline 3 & 71 & 45 \\
4 & 150 & 15 \\
5 & 188 & 2.5 \\
6 & 220 & 0.3 \\
7 & 231 & 0.03 \\
\hline
\end{tabular}

Parameters of the structure as in Fig. 4, but with increasing number of air slits per mirror.

corresponding cavity mode for the system with $N=7$ air slits between consecutive cavities is plotted in Fig. 8b. In Fig. 8c the imaginary part of the energy corresponding to the photonic modes of Fig. $8 \mathrm{~b}$ is shown. The vertical $Q$-factor of this cavity mode is calculated to be $Q_{\mathrm{v}}=1700$. It should be noted that the imaginary part of this cavity mode is roughly as dispersive as the one of Fig. 5b when looked on a linear scale (it seems more dispersive in Fig. 8c because it is an order of magnitude smaller and it is represented in a log scale). In Table 2 the FME results corresponding to the same parameters are presented. They lead again to conclusions similar to those of the previous case: the $Q$-factor from FME calculations increases with the number of air slits and tends to the $Q_{\mathrm{v}}$ obtained with the GME calculation, with a very low transmission coefficient. Transmission experiments performed on systems with first order Bragg mirrors cavity modes were reported in (Peyrade et al. 2002), and the present theoretical results are in good agreement with the experimental data.

In Fig. 9 we show the reflectance of the first-order cavity structure in the energy region of the defect mode. The spectral structure corresponding to the cavity mode is very narrow and has a non-negligible dispersion with the incidence angle, in agreement with the results obtained by the GME method
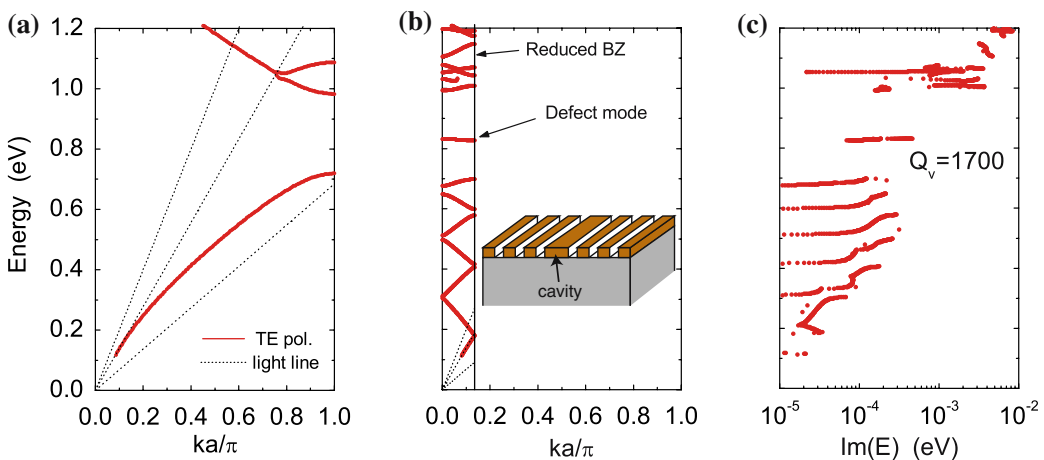

Fig. 8. (a) Photonic dispersion of a $1 \mathrm{D}$ lattice of Silicon stripes on a $\mathrm{SiO}_{2}$ cladding with lattice constant $a=310 \mathrm{~nm}$, thickness $d=260 \mathrm{~nm}$, air fraction $f_{\text {air }}=0.3226$. (b) 1 D cavity of length $L_{\text {cav }}=335 \mathrm{~nm}$ and seven air slits in the mirrors; (c) imaginary part of energy for the cavity system and vertical $Q$-factor. 
Table 2. Quality factors and transmission peaks calculated with the FME method

\begin{tabular}{lcl}
\hline Number of slits & $Q$-factor & $T_{\text {peak }}(\%)$ \\
\hline 3 & 79 & 90 \\
4 & 250 & 72 \\
5 & 625 & 38 \\
6 & 1071 & 10 \\
7 & 1500 & 2 \\
8 & 1580 & 0.13 \\
\hline
\end{tabular}

Parameters of the structure as in Fig. 8, with increasing number of air slits per mirror.

(see Fig. 8c). It is interesting to notice that the cavity mode becomes more pronounced at high values of the angle of incidence, close to the grazing condition. This follows from the fact that the defect mode associated with the first-order structure has the dominant wave vector components close to the edge of the BZ, i.e., below the light line. In other words, diffraction occurs mainly for a parallel wave vector close to the air light line. We conclude that the energies and $Q$-factors of cavity modes associated with both first- and second-order Bragg mirrors can be measured by surface reflectance in a periodic structure, however the cavity mode is more easily observed at large (small) values of the angle of incidence for the first-order (second-order) cavity structure.

As a final comment, the computing time and memory requirements are quite modest for the three methods employed in this work, for the present case of a $1 \mathrm{D}$ waveguide patterning. The largest computational effort is required by the SMM because of the need of calculating reflectance spectra from the surface at different angles of incidence. Anyway, also in the latter case the computing time is of the order of a few minutes on a Personal workstation.

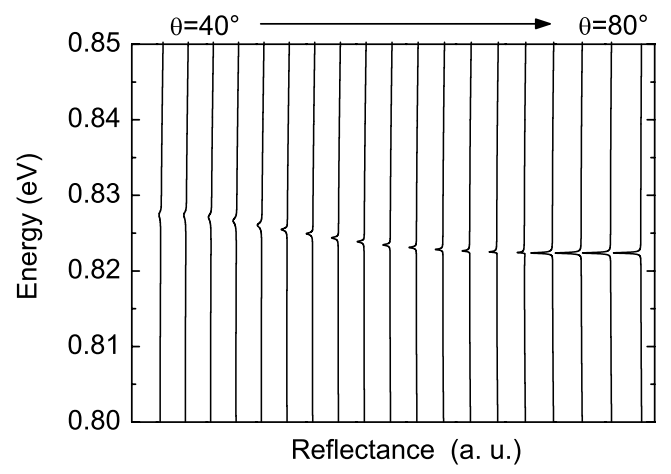

Fig. 9. Reflectance of a cavity in a first-order Bragg structure (same parameters as in Fig. 8), showing the resonant structure corresponding to the cavity mode. Reflectance spectra are calculated from $40^{\circ}$ to $85^{\circ}$ in steps of $2.5^{\circ}$ and are shifted by $\Delta R=0.5$ for clarity. 


\section{Conclusion}

We have presented numerical results for cavity modes in 1D photonic crystal slabs based on SOI technology. The three methods employed in this work, namely guided-mode expansion, grating theory or scattering-matrix method, and Fourier modal expansion have been shown to lead to the same values for the cavity $Q$-factors. Cavity structures with first-order Bragg mirrors have higher $Q$-factors than structures with second-order mirrors. The GME and SMM methods treat systems with a periodic repetition of the cavity along the 1D axis and infinite extension in the vertical direction, while the FME method deals with a finite structure along the 1D axis and perfectly matched layers in the vertical direction. The $Q$-factors obtained by the FME method increase with the number of periods in the Bragg mirrors and tend to the vertical $Q$ determined by GME or SMM methods when the effect of escape in the finite 1D structure is negligible. Thus, the $Q$-factor of cavity modes can be obtained from experiments by coupling from the surface of the waveguide and measuring the reflectance of a periodized cavity system. The cavity mode in reflectance spectra is more easily detectable close to grazing (normal) incidence for structures with first- (second-) order mirrors. This may be advantageous over transmission measurements that are made difficult by the length of the structure, when the transmission in the cavity mode is very low owing to diffraction losses.

\section{References}

Andreani, L.C. and M. Agio. IEEE J. Quantum Electron 38 891, 2002.

Andreani, L.C. Phys. Stat. Sol. (b) 234 139, 2002.

Andreani, L.C. and M. Agio. Appl. Phys. Lett. 82 2011, 2003.

Astratov, V.N., D.M. Whittaker, I.S. Culshaw, R.M. Stevenson, M.S. Skolnick, T.F. Krauss and R.M. De La Rue. Phys. Rev. B 60 R16255, 1999.

Berenger, J.P. J. Comput. Phys. 114 185, 1994.

Bristow, A.D., D.M. Whittaker, V.N. Astratov, M.S. Skolnick, A. Tahraoui, T.F. Krauss, M. Hopkinson, M.P. Croucher and G.A. Gehring. Phys. Rev. B $68033303,2003$.

Cao, Q., P. Lalanne and J.P. Hugonin. J. Opt. Soc. Am. A 19 335, 2002.

Čtyroký, J., S. Helfert, R. Pregla, P. Bienstman, R. Baets, R. de Ridder, R. Stoffer, G. Klaase, J. Petráček, P. Lalanne, J.P. Hugonin and R.M. De La Rue. Opt. Quantum Electron. 34 455, 2002.

Fujita, T., Y. Sato, T. Kuitani and T. Ishihara. Phys. Rev. B 57 12428, 1998.

Galli, M., M. Belotti, D. Bajoni, M. Patrini, G. Guizzetti, D. Gerace, M. Agio, L.C. Andreani and Y. Chen. Phys. Rev. B $70081307,2004$.

Gerace, D. and L.C. Andreani. Phys. Rev. E 69 056603, 2004a.

Gerace, D. and L.C. Andreani. Opt. Lett. 29 1897, 2004 b.

Ho, K.M., C.T. Chan and C.M. Soukoulis. Phys. Rev. Lett. 65 3152, 1990.

Johnson, S.J., S. Fan, P.R. Villeneuve, J.D. Joannopoulos and L.A. Kolodziejski. Phys. Rev. B 605751 , 1999.

Johnson, S.J. and J.D. Joannopoulos. Photonic Crystals: the Road from Theory to Practice, Kluwer, Dordrecht, 2002. 
Lalanne, P. and G.M. Morris. J. Opt. Soc. Am. A 13 779, 1996.

Lalanne, P. and E. Silberstein. Opt. Lett. 25 1092, 2000.

Lalanne, P. IEEE J. Quantum Electron. 38 800, 2002.

Lalanne, P. and J.P. Hugonin. IEEE J. Quantum Electron. 39 1430, 2003.

Lalanne, P., J.P. Hugonin and J.M. Gérard. Appl. Phys. Lett. 84 4726, 2004a.

Lalanne, P., S. Mias and J.P. Hugonin. Opt. Exp. 12 458, 2004 b.

Li, L. J. Opt. Soc. Am. A 14 2758, 1997.

Moharam, M.G., E.B. Grann, D.A. Pommet and T.K. Gaylord. J. Opt. Soc. Am. A 12 1068, 1995.

Ochiai, T. and K. Sakoda. Phys. Rev. B 63 125107, 2001a.

Ochiai, T. and K. Sakoda. Phys. Rev. B 64 045108, 2001 b.

Painter, O., J. Vučković and A. Scherer. J. Opt. Soc. Am. B 16 275, 1999.

Palamaru, M. and P. Lalanne. Appl. Phys. Lett. 78 1466, 2001.

Patrini, M., M. Galli, F. Marabelli, M. Agio, L.C. Andreani, D. Peyrade and Y. Chen. IEEE J. Quantum Electron 38 885, 2002.

Peyrade, D., Y. Chen, A. Talneau, M. Patrini, M. Galli, F. Marabelli, M. Agio, L.C. Andreani, E. Silberstein and P. Lalanne. Microelectron. Engin. 61-62 529, 2002.

Popov, E. Prog. Optics 31 141, 1993.

Popov, E. and M. Nevière. J. Opt. Soc. Am. A 17 1773, 2000.

Sakoda, K. Optical Properties of Photonic Crystals, Springer, Berlin, 2001.

Sauvan, C., P. Lalanne, J.C. Rodier, J.P. Hugonin and A. Talneau. IEEE Photon. Technol. Lett. 15 1243, 2003.

Silberstein, E., P. Lalanne, J.P. Hugonin and Q. Cao. J. Opt. Soc. Am. A 18 2865, 2001.

Whittaker, D.M. and I.S. Culshaw. Phys. Rev. B 60 2610, 1999.

Yariv, A. and P. Yeh. Optical Waves in Crystals, Wiley, New York, 1984. 